\title{
BOUNDARY AT INFINITY OF SYMMETRIC RANK ONE SPACES
}

\author{
S. BUYALO AND A. KUZNETSOV
}

\begin{abstract}
It is shown that the canonical Carnot-Carathéodory spherical and horospherical metrics, which are defined on the boundary at infinity of every rank one symmetric space of noncompact type, are visual; i.e., they are bi-Lipschitz equivalent with universal bi-Lipschitz constants to the inverse exponent of Gromov products based in the space and on the boundary at infinity respectively.
\end{abstract}

\section{§1. INTRODUCTION}

Let $M$ be a rank one symmetric space of noncompact type. There are two classes of natural metrics on the boundary at infinity $\partial_{\infty} M$. First, these are spherical and horospherical metrics. Given $o \in M$, for every $t>0$ we consider the Riemannian metric $d s_{t}^{2}$ induced from $M$ on the sphere $S_{t} \subset M$ of radius $t$ centered at $o$. Identifying $S_{t}$, $\partial_{\infty} M$ with the unit sphere $U_{o} M \subset T_{o} M$ via the radial projection from $o$, we view $d s_{t}^{2}$ as the Riemannian metric on $U_{o} M$ for all $t>0$. Then there exists a limit

$$
d s_{\infty}=\lim _{t \rightarrow \infty} e^{-t} d s_{t},
$$

which is a Carnot-Carathéodory metric. The respective (bounded) interior distance is the spherical metric $d_{\infty}$ on $\partial_{\infty} M$ based at $o$.

Similarly, given a Busemann function $b: M \rightarrow \mathbb{R}$ centered at $\omega \in \partial_{\infty} M$, for every $t>0$ we can consider the Riemannian metric $d s_{b, t}^{2}$ induced from $M$ on the horosphere $H_{b, t}=b^{-1}(t)$. Identifying $H_{b, t}, \partial_{\infty} M \backslash\{\omega\}$ with the fixed horosphere $H=H_{b, 0}$ via the radial projection from $\omega$, we view $d s_{b, t}^{2}$ as the Riemannian metric on $H$ for all $t \in \mathbb{R}$. Then there exists a limit

$$
d s_{b}=\lim _{t \rightarrow \infty} e^{-t} d s_{b, t}
$$

which is a Carnot-Carathéodory metric. The respective (unbounded) interior distance is the horospherical metric $d_{b}$ on $\partial_{\infty} M \backslash\{\omega\}$ associated with $b$.

Second, since $M$ is a CAT(-1)-space, in particular, $M$ is Gromov hyperbolic, there are visibility metrics on the Gromov boundary at infinity of $M$. This boundary coincides with the geodesic boundary $\partial_{\infty} M$, and for $\xi, \eta \in \partial_{\infty} M$ we have the Gromov product $(\xi \mid \eta)_{o}$ based at $o$, and similarly, for $\xi, \eta \in \partial_{\infty} M \backslash\{\omega\}$ we have the Gromov product $(\xi \mid \eta)_{b}$ associated with a Busemann function $b$. Any (bounded) metric on $\partial_{\infty} M$ that is bi-Lipschitz equivalent to the function $(\xi, \eta) \rightarrow a^{-(\xi \mid \eta)_{o}}$, and any (unbounded) metric on $\partial_{\infty} M \backslash\{\omega\}$ that is bi-Lipschitz equivalent to the function $(\xi, \eta) \rightarrow a^{-(\xi \mid \eta)_{b}}$ for some $a>1$, are called visibility metrics.

These two classes of metrics are certainly well known, but surprisingly, we did not find in the literature an answer to the natural question as to how they are related (except for

2010 Mathematics Subject Classification. Primary 53C23.

Key words and phrases. Carnot-Carathéodory spherical metric, Carnot-Carathédory horospherical metric, visual metric, Gromov product.

Supported by RFBR (grant no. 08-01-00079a). 
the case where $M=\mathrm{H}^{n}$ is real hyperbolic). Our aim in this paper is to fill this gap. We obtain the following.

Theorem 1.1. There are constants $c_{1}, c_{2}>0$ such that for every symmetric rank one space $M$ of noncompact type, the horospherical metric $d_{b}$ on $\partial_{\infty} M \backslash\{\omega\}$ associated with an arbitrary Busemann function $b: M \rightarrow \mathbb{R}$ centered at any point $\omega \in \partial_{\infty} M$ satisfies

$$
c_{1} e^{-(\xi \mid \eta)_{b}} \leq d_{b}(\xi, \eta) \leq c_{2} e^{-(\xi \mid \eta)_{b}}
$$

for each $\xi, \eta \in \partial_{\infty} M \backslash\{\omega\}$.

Theorem 1.2. There are constants $c_{1}, c_{2}>0$ such that for every symmetric rank one space $M$ of noncompact type, the spherical metric $d_{\infty}$ on $\partial_{\infty} M$ based at any o $\in M$ satisfies

for each $\xi, \eta \in \partial_{\infty} M$.

$$
c_{1} e^{-(\xi \mid \eta)_{o}} \leq d_{\infty}(\xi, \eta) \leq c_{2} e^{-(\xi \mid \eta)_{o}}
$$

Remark 1.3. In the case where $M=\mathrm{H}^{n}, n \geq 2$, is real hyperbolic, the metric $2 d_{\infty}$ is isometric to the standard metric of the unit sphere $S^{n-1} \subset \mathbb{R}^{n}$ for every base point $o \in \mathrm{H}^{n}$. On the other hand, an easy argument shows (see [BS, sect. 2.4.3]) that the function $(\xi, \eta) \mapsto e^{-(\xi \mid \eta)_{o}}$ coincides with half of the chordal metric,

$$
e^{-(\xi \mid \eta)_{o}}=\frac{1}{2}|\xi-\eta|
$$

for every $\xi, \eta \in S^{n-1}$.

Similarly, for every Busemann function $b: \mathrm{H}^{n} \rightarrow \mathbb{R}$ centered at $\omega \in \partial_{\infty} \mathrm{H}^{n}$, the horospherical metric $d_{b}$ is isometric to the standard $\mathbb{R}^{n-1}$, and an easy calculation in the upper half-space model shows that $d_{b}(\xi, \eta)=e^{-(\xi \mid \eta)_{b}}$ for every $\xi, \eta \in \partial_{\infty} \mathrm{H}^{n} \backslash\{\omega\}$.

Remark 1.4. For the case where $M=\mathbb{C} H^{2}$ is the complex hyperbolic plane, a weaker version of Theorem 1.2 was obtained in $\mathrm{Ku}$.

Remark 1.5. In $\left[\mathrm{BO}\right.$ it was proved that the function $\left(u, u^{\prime}\right) \mapsto\left|u u^{\prime}\right|:=e^{-\left(u \mid u^{\prime}\right)_{o}}$ is a metric on the boundary at infinity $\partial_{\infty} X$ of any CAT(-1)-space $X$ for every $o \in X$. Moreover, in [FS] it was proved that $\partial_{\infty} X$ endowed with this metric is a Ptolemy metric space; i.e., the Ptolemy inequality $|x y| \cdot|u v| \leq|x u| \cdot|y v|+|x v| \cdot|y u|$ holds true for all $x$, $y, u, v \in \partial_{\infty} X$.

\section{§2. Preliminaries}

2.1. Symmetric rank one spaces. Every rank one symmetric space $M$ of noncompact type is a hyperbolic space $\mathbb{K} H^{n}$ over the real numbers $\mathbb{K}=\mathbb{R}$, or the complex numbers $\mathbb{K}=\mathbb{C}$, or the quaternions $\mathbb{K}=\mathbb{H}$, or the octonions $\mathbb{K}=\mathbb{C} a$, and $\operatorname{dim} M=n \cdot \operatorname{dim} \mathbb{K}$, where $n \geq 2$ and $n=2$ in the case where $\mathbb{K}=\mathbb{C} a$ (see, e.g., Wo ). We use the standard notation $T M$ for the tangent bundle of $M$ and $U M$ for the subbundle of unit vectors.

2.1.1. Curvature operator. One of the equivalent characterizations of the Riemannian symmetric spaces is that their curvature tensor is parallel, $\nabla \mathcal{R}=0$; see, e.g., Wo. It follows that for every unit vector $u \in U_{o} M$, where $o \in M$, the eigenspaces $E_{u}(\lambda)$ of the curvature operator $\mathcal{R}(\cdot, u) u: u^{\perp} \rightarrow u^{\perp}$, where $u^{\perp} \subset T_{o} M$ is the subspace orthogonal to $u$, are parallel along the geodesic $\gamma(t)=\exp _{o}(t u), t \in \mathbb{R}$, and the respective eigenvalues $\lambda$ are constant along $\gamma$. Note that $u^{\perp}$ is identified with the tangent space $T_{u}\left(U_{o} M\right)$ of the unit sphere $U_{o} M$ at $u$.

If $M=\mathbb{K} \mathrm{H}^{n}, n \geq 2$ ( $n=2$ in the case of octonions), then the eigenvalues of the curvature operator $\mathcal{R}(\cdot, u) u$ are $\lambda=-1,-4$. The dimensions of the respective eigenspaces are $\operatorname{dim} E_{u}(-1)=(n-1) \operatorname{dim} \mathbb{K}, \operatorname{dim} E_{u}(-4)=\operatorname{dim} \mathbb{K}-1, u^{\perp}=E_{u}(-1) \oplus E_{u}(-4)$. In 
view of Remark 1.3 , we assume that $\mathbb{K} \neq \mathbb{R}$. Then $E_{u}(-4) \neq\{0\}$ and the sectional curvatures of $M$ are pinched, $-4 \leq K_{\sigma} \leq-1$. We denote by $E(\lambda)$ the subbundle of the tangent bundle $T\left(U_{o} M\right)$ with fibers $E_{u}(\lambda), u \in U_{o} M, \lambda=-1,-4$.

2.2. Boundary at infinity and Carnot-Carathéodory metrics. The (geodesic) boundary at infinity $\partial_{\infty} M$ of $M$ consists of the equivalence classes of geodesic rays in $M$, where two rays are equivalent if they are at a finite Hausdorff distance from each other, and it is equipped with the standard (or cone) topology. In this topology, the map $r_{o}: U_{o} M \rightarrow \partial_{\infty} M$ that takes $u \in U_{o} M$ to the class of the geodesic rays $\exp _{o}(t u), t \geq 0$, is a homeomorphism for every $o \in M$.

2.2.1. Busemann functions and horospheres. The Busemann function $b=b_{\gamma}: M \rightarrow \mathbb{R}$ associated with a geodesic ray $\gamma \in \omega \in \partial_{\infty} M$ is defined by $b(x)=\lim _{t \rightarrow \infty}(|x \gamma(t)|-t)$. In this case, we say that $b$ is centered at $\omega$. We denote by $\mathcal{B}(\omega)$ the set of all Busemann functions centered at $\omega$. Two functions $b, b^{\prime} \in \mathcal{B}(\omega)$ differ by a constant, and moreover, $\mathcal{B}(\omega)$ is parametrized by the values of $b \in \mathcal{B}(\omega)$ at a fixed point $o \in M$, which can be arbitrary.

For $b \in \mathcal{B}(\omega), t \in \mathbb{R}$, the horosphere $H_{b, t}=b^{-1}(t)$ is a smooth hypersurface in $M$. Again, we denote by $E(\lambda)$ the subbundle of the tangent bundle $T H_{b, t}$, the fiber of which at every point $x \in H_{b, t}$ is the eigenspace $E_{u}(\lambda)$ of the curvature operator $\mathcal{R}(\cdot, u) u$, where $u=\operatorname{grad} b(x), \lambda=-1,-4$. We denote by $r_{b, t}: H_{b, t} \rightarrow \partial_{\infty} M \backslash\{\omega\}$ the radial projection map that takes every $x \in H_{b, t}$ to the class $\gamma(\infty) \in \partial_{\infty} M$, where $\gamma: \mathbb{R} \rightarrow M$ is a (unique) geodesic with $\gamma(-\infty)=\omega$ passing through $x$. Then $r_{b, t}$ is a homeomorphism.

We denote by $\rho_{o}: M \backslash\{o\} \cup \partial_{\infty} M \rightarrow U_{o} M$ the radial projection; $\rho_{o}(x)$ is the unit vector tangent to the geodesic $o x$ at $o$.

Lemma 2.1. For any $o \in M, \omega \in \partial_{\infty} M$, and $b \in \mathcal{B}(\omega)$, the composition $f=\rho_{o} \circ r_{b, 0}$ : $H=H_{b, 0} \rightarrow U_{o} M$ is a Lipschitz embedding; its differential df is well defined on the $E(-1)$-subbundle of $T H$, preserves the $E(-1)$-subbundles of the tangent bundles $T H$ and $T\left(U_{o} M\right)$, and is conformal on them.

Proof. The composition $f_{s}: H \rightarrow U_{o} M$ of the radial projection $H \rightarrow H_{b, s}$ from $\omega$ with $\rho_{o}$ is a smooth embedding for all sufficiently large $s \in \mathbb{R}$, which approximates $f$ as $s \rightarrow \infty$. We show that the differential $d_{x} f_{s}$ is uniformly bounded in $s$ for every $x \in H$ and almost preserves the $E(-1)$-subbundles as $s \rightarrow \infty$.

For $x \in H$ and $v \in E_{u}(\lambda) \subset T_{x} H, u=\operatorname{grad} b(x)$, there is a unique Jacobi vector field $V$ along the geodesic $\gamma: \mathbb{R} \rightarrow M, \gamma(-\infty)=\omega, \gamma(0)=x$, such that $V(0)=v$ and $V(s) \rightarrow 0$ as $s \rightarrow-\infty$. The direction field $V /|V|$ is parallel along $\gamma$, and $|V(s)|=e^{s \sqrt{|\lambda|}}|v|$.

For $s \in \mathbb{R}$, we put $\alpha_{s}=\angle_{\gamma(s)}(o, \omega), \beta_{s}=\angle_{o}(\omega, \gamma(s)), \tau_{s}=|o \gamma(s)|$. Then

$$
\alpha_{s} \leq 4 e^{-\tau_{s}} / \beta_{s},
$$

by comparison with $\mathrm{H}^{2}$, and the (generalized) formula for the parallelism angle. Note that $\beta_{s} \rightarrow L_{o}(\omega, \gamma(\infty)) \neq 0$ as $s \rightarrow \infty$ for all $x \in H$. Furthermore, $\lim _{s \rightarrow \infty}\left(\tau_{s}-s\right)=b_{\gamma_{x}}(o)$, where $\gamma_{x}=x \gamma(\infty)$. Thus, $\tau_{s}=s+b_{\gamma_{x}}(o)+o(1)$ as $s \rightarrow \infty$.

For $w=d f_{s}(v) \in T\left(U_{o} M\right)$, let $W$ be the Jacobi field along $o \gamma(s)$ with the initial data $W(o)=0, \dot{W}(o)=w$. Then, by the definition of $w$, the vector $W(\gamma(s))$ is the orthogonal projection of $V(s)$ to $u^{\prime \perp}$, where $u^{\prime} \in U_{\gamma(s)} M$ is tangent to the segment $o \gamma(s)$. The angle between $V(s)$ and $W(\gamma(s))$ is at most $\alpha_{s}$. Thus, $\left(1-\alpha_{s}^{2}\right)|V(s)| \leq|W(\gamma(s))| \leq|V(s)|$.

In the orthogonal decomposition $W=W_{1}+W_{2}$ with respect to the eigenspaces of the curvature operator, the sections $W_{i}$ of $E\left(-i^{2}\right), i=1,2$, are Jacobi fields along $o \gamma(s)$. Also, we have $\left|W_{i}(\gamma(s))\right| \leq|W(\gamma(s))| \leq|V(s)|$. The eigenspaces of the curvature 
operator $\mathcal{R}(\cdot, u) u$ depend smoothly on the direction $u$, so that for $i=\sqrt{|\lambda|}$ the angle between $W(\gamma(s))$ and $W_{i}(\gamma(s))$ is $O\left(\alpha_{s}\right)$, whence $\left|W_{i}(\gamma(s))\right| \geq\left(1-O\left(\alpha_{s}\right)\right)|V(s)|$.

Since $\dot{W}=\dot{W}_{1}+\dot{W}_{2}$, we have $\left|W_{i}(\gamma(s))\right|=\frac{\sinh \left(i \tau_{s}\right)}{i}\left|w_{i}\right|$ for $i=1,2$, where $w=w_{1}+w_{2}$ is the orthogonal decomposition with respect to the eigenspaces of the curvature operator. Thus, for $i=\sqrt{|\lambda|}$ we have

$$
\left(1-O\left(\alpha_{s}\right)\right) i|V(s)| / \sinh \left(i \tau_{s}\right) \leq\left|w_{i}\right| \leq i|V(s)| / \sinh \left(i \tau_{s}\right),
$$

which implies that $\left|w_{i}\right| \sim 2 i e^{-i b_{\gamma_{x}}(o)}|v|$ as $s \rightarrow \infty$. Note that $b_{\gamma_{x}}(o) \geq-\operatorname{dist}(o, H)$ and $b_{\gamma_{x}}(o) \rightarrow \infty$ as $x \rightarrow \infty$ in $H$.

If $\lambda=-1$, then $\left|w_{2}\right| \leq 2|V(s)| / \sinh 2 \tau_{s} \leq 8 e^{-s} e^{-2 b_{\gamma_{x}}(o)}|v| \rightarrow 0$ as $s \rightarrow \infty$. This shows that $d f_{s}$ almost preserves the subbundle $E(-1)$ and is almost conformal on it with the factor $2 e^{-b_{\gamma_{x}}(o)}$.

It remains to consider the case where $\lambda=-4$. We already know that $\left|w_{2}\right| \sim$ $4 e^{-2 b_{\gamma_{x}}(o)}|v|$ as $s \rightarrow \infty$. On the other hand, $\left|W_{1}(\gamma(s))\right|=O\left(\alpha_{s}\right)|V(s)|$, whence $\left|w_{1}\right|=$ $\left|W_{1}(\gamma(s))\right| / \sinh \tau_{s} \leq O\left(\alpha_{s}\right) e^{2 s}|v| / \sinh \tau_{s}$. Using (11), we obtain $\left|w_{1}\right| \leq c e^{-2 b_{\gamma_{x}}(o)}|v|$ for some constant $c>0$ depending only on $x \in H, c=c(x)$. Thus, $\left|d_{x} f_{s}(v)\right| \leq\left|w_{1}\right|+\left|w_{2}\right| \leq$ $(c+4) e^{-2 b_{\gamma_{x}}(o)}|v|$, and hence, the norm $\left\|d_{x} f_{s}\right\|$ is uniformly bounded in $s$ for every $x \in H$. We conclude that $f=\lim _{s \rightarrow \infty} f_{s}$ is Lipschitz.

Remark 2.2. Lemma 2.1 is a refinement of [Pa, Lemme 9.6]. We have added the estimate of $d f_{s}$ on $E(-4)$ based on the estimate (1), which leads to the conclusion that the embedding $f: H \rightarrow U_{o} M$ is Lipschitz.

2.2.2. Isometries of $M$ and invariant metrics on a horosphere. Given $\omega \in \partial_{\infty} M$, there is a subgroup $N_{\omega}$ in the isometry group $G=$ Isom $M$ (a maximal unipotent subgroup) that leaves invariant every horosphere $H$ in $M$ centered at $\omega$ and is simply transitive on $H$ and on $\partial_{\infty} M \backslash\{\omega\}$. The group $N=N_{\omega}$ is a nilpotent Lie group of dimension $\operatorname{dim} N=\operatorname{dim} M-1$. If $M=\mathbb{C H}^{2}$, then $N$ is isomorphic to the classical Heisenberg group.

Fixing a base point $o \in H$, we identify $N$ with the orbit $H=N(o)$, and identify the tangent space $T_{o} H$ with the Lie algebra $\mathcal{N}$ of $N$. Then $N$ leaves invariant the subbundles $E(\lambda)$ of $T H$, and $\mathcal{N}=E_{1} \oplus E_{2}$, where $E_{i}=E_{u}\left(-i^{2}\right), i=1,2$, are the fibers of $E(\lambda)$ at $o$. Moreover, $E_{2}=\left[E_{1}, E_{1}\right]$ in $\mathcal{N}$; in particular, $\mathcal{N}$ is the minimal subalgebra in $\mathcal{N}$ containing $E_{1}$; see, e.g., $\mathrm{Pa}$.

The subbundle $E(-4) \subset T H$ is integrable, and the corresponding fibers $F$ are intersections of $\mathbb{K}$-lines with $H$, where each $\mathbb{K}$-line in $M$ is a totally geodesic subspace isometric to the hyperbolic space $\frac{1}{2} \mathrm{H}^{\operatorname{dim} \mathbb{K}}$ of constant curvature -4 ; see [Mo, $\left.\S 20\right]$ and Pa. Therefore, every fiber $F$ is flat in $H$. The corresponding fibration $\phi: H \rightarrow B$ is a Riemannian submersion (with respect to the induced Riemannian metric on $H$ ) with the base $B$ isometric to $\mathbb{R}^{(n-1) \operatorname{dim} \mathbb{K}}$. The group $N$ acts by isometries on $B$, and the kernel of that action is the center $Z$ of $N$, so that $N / Z=\mathbb{R}^{(n-1) \operatorname{dim} \mathbb{K}}$, and the submersion $\phi$ is equivariant with respect to these actions of $N$ on $H$ and $B$.

If $E_{1}$ is identified with $\mathbb{K}^{n-1}$ and $E_{2}$ with $\operatorname{Im} K$, then the Lie algebra structure of $\mathcal{N}=E_{1} \oplus E_{2}$ is given by $[v, w]=2 \operatorname{Im}(v, w)$ for $v, w \in E_{1}$, where $v=\left(v_{2}, \ldots, v_{n}\right)$ and $(v, w)=\sum_{i} \bar{v}_{i} w_{i}$; see $[\mathrm{Mo},(19.14)]$.

We call the subbundle $E=E(-1) \subset T H$ the polarization on the horosphere $H$. A piecewise smooth curve $\sigma: I \rightarrow H$ is said to be E-horizontal if $\dot{\sigma}(t) \in E$ for every $t \in I \subset \mathbb{R}$. Its length is $\ell(\sigma)=\int_{I}|\dot{\sigma}(t)| d t$. We define the distance $d_{E}\left(x, x^{\prime}\right)$ by taking the infimum of the lengths of $E$-horizontal curves between $x, x^{\prime} \in H$. This quantity is finite because $E_{1}$ generates $\mathcal{N}$, so that $E$ is completely nonintegrable. The restriction $d s_{E}^{2}=d s^{2} \mid E$ of the Riemannian metric $d s^{2}$ of $M$ will be called the Carnot-Carathéodory 
metric, and the corresponding distance $d_{E}$ is the Carnot-Carathéodory distance on $H$. Furthermore, since $d \phi$ is isometric on $E$, $\phi$ preserves the lengths of $E$-horizontal curves.

Let $d_{H}$ denote the interior distance on $H$ induced from $M$.

Lemma 2.3. We have

$$
\sup \frac{d_{E}^{2}(o, x)}{d_{H}(o, x)} \leq 17
$$

where the supremum is taken over all $x \in H$ with $|o x| \leq 1$ in $M$.

Proof. Let $F=\phi^{-1}(\phi(o))$ be the fiber of $\phi$ through $o$. First, we show that the ratio $r(x)=d_{E}^{2}(o, x) / d_{H}(o, x)$ is independent of $x \in F$.

For every $\lambda>0$ there is a homothety $h_{\lambda}: N \rightarrow N$ that is an automorphism of $N$ and that acts on $F$ as the homothety with coefficient $\lambda^{2}$ and on $B$ as the homothety with coefficient $\lambda$; see e.g. [Pa. In particular, $\ell\left(h_{\lambda}(\sigma)\right)=\lambda \ell(\sigma)$ for the length $\ell(\sigma)$ of every $E$-horizontal curve $\sigma \subset H$. It follows that $r\left(h_{\lambda}(x)\right)=r(x)$ for every $\lambda>0$. Next, the stabilizer $G_{o \omega} \subset G$ of $o \cup \omega$ acts transitively on the rays $o \xi \subset F$ (see [Mo $\left.\S 21\right]$ ). Thus, $r=r(x)$ is independent of $x \in F \backslash\{o\}$.

For any $v, w \in E_{1} \subset \mathcal{N}=T_{o} H$, the commutator $[\exp s v, \exp s w]=x_{s}$ lies in $F=$ $\exp E_{2}$ for every $s \geq 0$, and $[v, w]=\lim _{s \rightarrow 0} \frac{1}{s^{2}} o x_{s}$. Representing the commutator as the end-point of a broken geodesic $\sigma_{s}$ with four edges, we find that $\sigma_{s}$ is an $E$-horizontal curve of length $2 s(|v|+|w|)$ that connects $o$ and $x_{s}$. Thus, $d_{E}\left(o, x_{s}\right) \leq 2 s(|v|+|w|)$, while $d_{H}\left(o, x_{s}\right)=s^{2}|[v, w]|+o\left(s^{2}\right)$. Hence, $r \leq 4(|v|+|w|)^{2} /|[v, w]|$. Taking $v, w \in E_{1}=\mathbb{K}^{n-1}$ orthonormal, $v=(1,0, \ldots, 0)$ and $w=(j, 0, \ldots, 0)$, where $j$ is one of $\operatorname{dim} \mathbb{K}-1$ imaginary units, we find $[v, w]=2 j$. Therefore, $|v|=|w|=1,|[v, w]|=2$, and $r \leq 8$.

In the general case, for $x \in N$ with $|o x| \leq 1$ in $M$, there is an $E$-horizontal curve $\sigma$ that connects $o$ and $x^{\prime}$ so that $\phi \circ \sigma$ is a segment in $B$ and $x^{\prime} x \subset F$, where $F$ is the fiber of $\phi$ through $x$. We have $\left|x^{\prime} x\right| \leq|o x| \leq 1, d_{H}(o, x) \geq \ell(\sigma), d_{H}\left(x^{\prime}, x\right)$, and $\sigma$ lies in a totally real plane in $M$, which is a geodesic subspace isometric to $\mathrm{H}^{2}$. Moreover, $\sigma$ is a horocycle in $\mathrm{H}^{2}$, which yields $\ell(\sigma) \leq 2 \sinh \frac{1}{2}$, and also $d_{H}\left(x^{\prime}, x\right) \leq \sinh 1$; see Theorem 2.4 below. Then $d_{E}(o, x) \leq \ell(\sigma)+d_{E}\left(x^{\prime}, x\right)$, whence

$$
\frac{d_{E}^{2}(o, x)}{d_{H}(o, x)} \leq \frac{d_{E}^{2}\left(x^{\prime}, x\right)}{d_{H}\left(x^{\prime}, x\right)}+\ell(\sigma)+2 d_{E}\left(x^{\prime}, x\right) \leq r+2 \sinh (1 / 2)+2 \sqrt{r \sinh 1} \leq 17 .
$$

2.3. Negatively pinched Hadamard manifolds. Let $M$ be a Hadamard manifold with sectional curvatures $-b^{2} \leq K_{\sigma} \leq-a^{2}, a>0$. Assume that a horosphere $H \subset M$ is fixed. We denote by $\left|x x^{\prime}\right|$ the distance between $x, x^{\prime} \in H$ in $M$ and by $\left|x x^{\prime}\right|_{H}$ the induced interior distance in $H$. We shall use the following result from [HI, Theorem 4.6].

Theorem 2.4. For every $x, x^{\prime} \in H$ we have

$$
\frac{2}{a} \sinh \frac{a}{2}\left|x x^{\prime}\right| \leq\left|x x^{\prime}\right|_{H} \leq \frac{2}{b} \sinh \frac{b}{2}\left|x x^{\prime}\right| .
$$

2.4. Gromov hyperbolic spaces. Most of the facts on hyperbolic spaces needed for what follows, except for information about equiradial points of infinite triangles (see Subsection 2.4.2), can be found, e.g., in [BS. We discuss these facts for symmetric rank one spaces $M$, though all of them remain true for the general proper CAT(-1)-spaces $X$. In this case, the geodesic boundary at infinity $\partial_{\infty} X$ coincides with the Gromov boundary at infinity. Every CAT(-1)-space is boundary continuous; see [BS, 3.4.2]. This simplifies the definitions of the key notions essentially, compared with the general case of Gromov hyperbolic spaces. 
2.4.1. Gromov products and $\delta$-triples. The Gromov product of $x, x^{\prime} \in M$ with respect to $o \in M$ is $\left(x \mid x^{\prime}\right)_{o}=\frac{1}{2}\left(|x o|+\left|x^{\prime} o\right|-\left|x x^{\prime}\right|\right)$. This quantity is always nonnegative by the triangle inequality.

Being a CAT(-1)-space, the space $M$ is $\delta$-hyperbolic (in the sense of Gromov); that is, for every triangle $x y z \subset M$, if $y^{\prime} \in x y, z^{\prime} \in x z$, and $\left|x y^{\prime}\right|=\left|x z^{\prime}\right| \leq(y \mid z)_{x}$, then $\left|y^{\prime} z^{\prime}\right| \leq \delta$, with the constant $\delta \leq \delta_{\mathrm{H}^{2}}$. It is known that the hyperbolicity constant for the real hyperbolic plane $\mathrm{H}^{2}$ equals $\delta_{\mathrm{H}^{2}}=2 \ln \tau=0.9624 \ldots$, where $\tau$ is the golden ratio, $\tau^{2}=\tau+1 ;$ see $[\mathrm{BS}$.

The condition above implies that for every $o, x, y, z \in M$ the $\delta$-inequality is valid, $(x \mid y)_{o} \geq \min \left\{(x \mid z)_{o},(y \mid z)_{o}\right\}-\delta$ (the converse is also true but with different $\delta$ ). This inequality can be rewritten in terms of $\delta$-triples. A triple of real numbers $(a, b, c)$ is called a $\delta$-triple if the two smallest of these numbers differ by at most $\delta$. Then the $\delta$-inequality is equivalent to the fact that the numbers $(x \mid y)_{o},(x \mid z)_{o}$, and $(y \mid z)_{o}$ form a $\delta$-triple.

The Gromov product of $x, y \in M$ with respect to a Busemann function $b: M \rightarrow \mathbb{R}$ is defined by

$$
(x \mid y)_{b}=\frac{1}{2}(b(x)+b(y)-|x y|) .
$$

This product, contrary to the standard case $(x \mid y)_{o}$, may take arbitrary real values. Busemann functions $b$ and $b^{\prime}$ centered at one and the same point $\omega \in \partial_{\infty} M$ differ by a constant, $b-b^{\prime}=$ const. Therefore, the Gromov products with respect to $b, b^{\prime}$ differ by the same constant. As above, for every $x, y, z \in M$ the numbers $(x \mid y)_{b},(x \mid z)_{b}$, and $(y \mid z)_{b}$ form a $\delta$-triple.

The Gromov product of points $\xi, \eta \in \partial_{\infty} M$ in the boundary at infinity with respect to $o \in M$ is the limit

$$
(\xi \mid \eta)_{o}=\lim _{s, t \rightarrow \infty}(\gamma(s) \mid \rho(t))_{o},
$$

where $\gamma=o \xi$ and $\rho=o \eta$ are geodesic rays (the limit exists by the monotonicity of the Gromov product). Note that $(\xi \mid \xi)_{o}=\infty$ for every $\xi \in \partial_{\infty} M$. Again, for any pairwise distinct points $\xi, \eta, \zeta \in \partial_{\infty} M$, the numbers $(\xi \mid \eta)_{o},(\xi \mid \zeta)_{o}$, and $(\eta \mid \zeta)_{o}$ form a $\delta$-triple.

Finally, the Gromov product of $\xi, \eta \in \partial_{\infty} M \backslash\{\omega\}$ with respect to a Busemann function $b: M \rightarrow \mathbb{R}$ centered at $\omega \in \partial_{\infty} M$ is the limit

$$
(\xi \mid \eta)_{b}=\lim _{s, t \rightarrow \infty}(\gamma(s) \mid \rho(t))_{b},
$$

where $\gamma=\omega \xi, \rho=\omega \eta$ are geodesics in $M$ with $\gamma(\infty)=\xi, \rho(\infty)=\eta$ (the limit exists by the monotonicity of the Gromov product). For different Busemann functions $b, b^{\prime}$ : $M \rightarrow \mathbb{R}$ with one and the same center $\omega$, the Gromov products $(\xi \mid \eta)_{b}$ and $(\xi \mid \eta)_{b^{\prime}}$ differ by a constant, $(\xi \mid \eta)_{b}-(\xi \mid \eta)_{b^{\prime}}=$ const for every $\xi, \eta \in \partial_{\infty} M \backslash\{\omega\}$. For any pairwise distinct points $\xi, \eta, \zeta \in \partial_{\infty} M \backslash\{\omega\}$, the numbers $(\xi \mid \eta)_{b},(\xi \mid \zeta)_{b}$ and $(\eta \mid \zeta)_{b}$ form a $\delta$-triple.

2.4.2. Equiradial points of a triangle. For every triangle $x y z \subset M$, there are three spheres centered at its vertices that are pairwise tangent to each other from outside. The tangent points lie on the sides of the triangle and are called the equiradial points. The collection of the equiradial points $u \in y z, v \in x z, w \in x y$ is uniquely determined by the conditions $|u z|=|v z|=(x \mid y)_{z},|u y|=|w y|=(x \mid z)_{y}$, and $|v x|=|w x|=(y \mid z)_{x}$.

If instead of spheres we take horospheres centered at vertices at infinity, then we obtain the definition of equiradial points of an infinite triangle in $M$. In this case, the existence of equiradial points and their relationship with the corresponding Gromov products is not completely obvious.

Proposition 2.5. For any pairwise distinct points $\xi, \eta, \zeta \in \partial_{\infty} M$, the infinite triangle $\xi \eta \zeta$ in $M$ possesses a unique collection of equiradial points $u \in \eta \zeta, v \in \xi \zeta, w \in \xi \eta$. 
Moreover, we have $(\eta \mid \zeta)_{b}=b(v)=b(w)$ for every $b \in \mathcal{B}(\xi),(\xi \mid \zeta)_{b^{\prime}}=b^{\prime}(u)=b^{\prime}(w)$ for every $b^{\prime} \in \mathcal{B}(\eta)$, and $(\xi \mid \eta)_{b^{\prime \prime}}=b^{\prime \prime}(u)=b^{\prime \prime}(v)$ for every $b^{\prime \prime} \in \mathcal{B}(\zeta)$.

Proof. There are uniquely determined points $v \in \xi \zeta$ and $w \in \xi \eta$ such that $(\eta \mid \zeta)_{b}=$ $b(v)=b(w)$ for every $b \in \mathcal{B}(\xi)$. We take a function $b$ so that $(\eta \mid \zeta)_{b}=0$. Next, we fix a function $b^{\prime} \in \mathcal{B}(\zeta)$ such that $b^{\prime}(v)=0$ and let $u=\eta \zeta \cap b^{\prime-1}(0)$. After that we fix a function $b^{\prime \prime} \in \mathcal{B}(\eta)$ such that $b^{\prime \prime}(u)=0$ and let $w^{\prime}=\xi \eta \cap b^{\prime \prime-1}(0)$. We show that $b\left(w^{\prime}\right)=0$, i.e., $w^{\prime}=w$.

Denote by $\eta(t) \zeta(t) \subset \eta \zeta$ the segment of length $2 t$ centered at $u$. Let $\eta_{\xi}:[0, \infty) \rightarrow \xi \eta$ be the natural parametrization of the ray $w^{\prime} \eta, \eta_{\xi}(0)=w^{\prime}, \eta_{\xi}(\infty)=\eta$, and let $\zeta_{\xi}$ : $[0, \infty) \rightarrow \xi \zeta$ be the natural parametrization of the ray $v \zeta, \zeta_{\xi}(0)=v, \zeta_{\xi}(\infty)=\zeta$. Since $b^{\prime}(u)=b^{\prime}(v)=0=b(v)$ and $b^{\prime \prime}\left(w^{\prime}\right)=b^{\prime \prime}(u)=0$, we have $b\left(\zeta_{\xi}(t)\right)=t, b\left(\eta_{\xi}(t)\right)=b\left(w^{\prime}\right)+t$, and ||$\eta_{\xi}(t) \zeta_{\xi}(t)|-| \eta(t) \zeta(t)||=o(1)$ as $t \rightarrow \infty$. Therefore,

$$
\begin{aligned}
0=(\eta \mid \zeta)_{b}=\lim _{t \rightarrow \infty}\left(\eta_{\xi}(t) \mid \zeta_{\xi}(t)\right)_{b} & =\frac{1}{2} \lim _{t \rightarrow \infty}\left(b\left(\eta_{\xi}(t)\right)+b\left(\zeta_{\xi}(t)\right)-\left|\eta_{\xi}(t) \zeta_{\xi}(t)\right|\right) \\
& =\frac{1}{2} \lim _{t \rightarrow \infty}\left(b\left(\eta_{\xi}(t)\right)-t+b\left(\zeta_{\xi}(t)\right)-t+o(1)\right) \\
& =b\left(w^{\prime}\right) / 2
\end{aligned}
$$

This means that the horospheres $b^{-1}(0), b^{-1}(0), b^{\prime \prime-1}(0)$ centered at $\xi, \eta, \zeta$, respectively, touch each other from outside; i.e., the points $u, v, w$ are equiradial for the infinite triangle $\xi \eta \zeta$.

Since $(\eta \mid \zeta)_{b}=b(v)=b(w)$ and the equiradial points are determined uniquely, we also have $(\xi \mid \eta)_{b^{\prime}}=b^{\prime}(u)=b^{\prime}(v),(\xi \mid \zeta)_{b^{\prime \prime}}=b^{\prime \prime}(u)=b^{\prime \prime}(w)$.

Remark 2.6. The equiradial points of infinite triangles in $M$ with one or two vertices in $M$ also exist and are determined uniquely, and the corresponding identities for the Gromov products are also valid for them. This can be proved by similar arguments.

Lemma 2.7. Let $u \in \eta \zeta, v \in \xi \zeta, w \in \xi \eta$ be the equiradial points of an infinite triangle in $M$ with pairwise distinct vertices $\xi, \eta, \zeta \in \partial_{\infty} M$. Then $|u v|,|u w|,|v w| \leq \delta$, where $\delta>0$ is the hyperbolicity constant for $M$.

Proof. The equiradial points of every finite triangle in $M$ are at a distance of at most $\delta$ from each other by the definition of $\delta$-hyperbolicity. Thus, it suffices to find a family of finite triangles in $M$, the collections of equiradial points of which approximate the collection $u, v, w$.

For $t>0$, consider $x \in v \xi, y \in w \eta, z \in u \zeta$ lying at a distance $t$ from the vertices of the rays, $|x v|=|y w|=|z u|=t$. As $t \rightarrow \infty$, we have $w x \rightarrow w \xi$, uy $\rightarrow u \eta, v z \rightarrow v \zeta$, and each of the distances $|w x|,|u y|,|v z|$ differs from $t$ by $o(1)$. Thus, each of the distances $|y x|,|z y|,|x z|$ differs from $2 t$ by $o(1)$. Hence, the Gromov products $(y \mid z)_{x},(x \mid z)_{y},(x \mid y)_{z}$ all are equal to $t$ up to $o(1)$. This immediately implies that the equiradial points of $x y z$ approximate the points $u, v, w$ as $t \rightarrow \infty$.

Lemma 2.8. Let $u \in \eta \zeta, v \in \xi \zeta, w \in \xi \eta$ be the equiradial points of an infinite triangle $\xi \eta \zeta \subset M$ with pairwise distinct vertices $\xi, \eta, \zeta \in \partial_{\infty} M$. Then for points $w^{\prime} \in w \eta$ and $v^{\prime} \in v \zeta$ with $\left|w^{\prime} w\right|=1+\delta=\left|v^{\prime} v\right|$, where $\delta$ is the hyperbolicity constant of $M$, we have $\left|v^{\prime} w^{\prime}\right| \geq 2$.

Proof. We take $w^{\prime \prime} \in u \eta$ and $v^{\prime \prime} \in u \zeta$ so that $\left|w^{\prime \prime} u\right|=1+\delta=\left|v^{\prime \prime} u\right|$. Then Lemma 2.7 shows that $\left|v^{\prime} v^{\prime \prime}\right| \leq \delta$ and $\left|w^{\prime} w^{\prime \prime}\right| \leq \delta$. Thus, $\left|v^{\prime} w^{\prime}\right| \geq\left|v^{\prime \prime} w^{\prime \prime}\right|-2 \delta=2$. 


\section{§3. Proof of Theorem 1.1}

Let $\delta$ denote the hyperbolicity constant of $M$; we have $\frac{1}{2} \delta_{\mathrm{H}^{2}} \leq \delta \leq \delta_{\mathrm{H}^{2}}<1$ by comparison with $\mathrm{H}^{2}$ and $\frac{1}{2} \mathrm{H}^{2}$. We fix $o \in M, \omega \in \partial_{\infty} M$, and a Busemann function $b \in \mathcal{B}(\omega)$ with $b(o)=0$. For $t \in \mathbb{R}$ we denote by $H_{b, t}=b^{-1}(t)$ the horosphere in $M$; let $H=H_{b, 0}$, and let $d s_{b, t}^{2}$ be the Riemannian metric on $H_{b, t}$ induced from $M$. We identify $H_{b, t}, \partial_{\infty} M \backslash\{\omega\}$ with $H$ via the radial projection from $\omega$ and view $d s_{b, t}^{2}$ as the Riemannian metric on $H$ for all $t \in \mathbb{R}$. Then the limit

$$
d s_{b}=\lim _{t \rightarrow \infty} e^{-t} d s_{b, t}
$$

exists and coincides with the Carnot-Carathéodory metric $d s_{E}$.

Indeed, for every $x \in H$ and $v \in E_{u}(\lambda) \subset T_{x} H$, where $u=\operatorname{grad} b(x)$, a unique Jacobi field $V$ along the geodesic $\exp _{x} t u, t \in \mathbb{R}$, with $V(t) \rightarrow 0$ as $t \rightarrow \infty$ has the parallel direction field $V /|V|$, and $|V(t)|=e^{t \sqrt{|\lambda|}}|v|$. Thus, $e^{-t} d s_{b, t}(v)=e^{(\sqrt{|\lambda|}-1) t}|v|$, and for $v \in E=E(-1)$ we have $e^{-t} d s_{b, t}(v)=|v|=d s_{b}(v)$ for all $t \in \mathbb{R}$, while $d s_{b}(v)=\infty$ for all nonzero $v \in E(-4)$.

We view the corresponding Carnot-Carathéodory distance $d_{b}$ as a metric on $\partial_{\infty} M \backslash\{\omega\}$; this metric is said to be horospherical.

Lemma 3.1. For each $\xi, \eta \in \partial_{\infty} M \backslash\{\omega\}$, we have

$$
d_{b}(\xi, \eta) \geq c_{1} e^{-(\xi \mid \eta)_{b}},
$$

where $c_{1}=2 e^{-(1+\delta)}$.

Proof. We assume that $\xi \neq \eta$, because otherwise there is nothing to prove. For $\varepsilon>0$, we take an $E$-horizontal curve $\sigma_{\infty} \subset \partial_{\infty} M \backslash\{\omega\}$ between $\xi$ and $\eta$ with length $\ell_{b}\left(\sigma_{\infty}\right) \leq$ $d_{b}(\xi, \eta)+\varepsilon$. Let $\sigma_{t} \subset H_{b, t}$ be its radial projection from $\omega$. Since the curve $\sigma_{t}$ connects the points $u_{t} \in \omega \eta$ and $v_{t} \in \omega \xi$ with $b\left(u_{t}\right)=b\left(v_{t}\right)=t$ and also is $E$-horizontal on $H_{b, t}$, its length $\ell_{b, t}\left(\sigma_{t}\right)$ is equal to $e^{t} \ell_{b}\left(\sigma_{\infty}\right)$.

Let $u \in \omega \eta$ and $v \in \omega \xi$ be the equiradial points of the triangle $\omega \xi \eta$. Then $t_{0}:=$ $(\xi \mid \eta)_{b}=b(u)=b(v)$. We put $t=t_{0}+1+\delta$. For $u_{t} \in \omega \eta, v_{t} \in \omega \xi$ we have $\left|u_{t} v_{t}\right| \geq 2$ by Lemma 2.8. Then $\ell_{b, t}\left(\sigma_{t}\right) \geq\left|u_{t} v_{t}\right| \geq 2$ and

$$
d_{b}(\xi, \eta)+\varepsilon \geq \ell_{b}\left(\sigma_{\infty}\right)=e^{-t} \ell_{b, t}\left(\sigma_{t}\right) \geq c_{1} e^{-(\xi \mid \eta)_{b}} .
$$

Since $\varepsilon$ has been chosen arbitrarily, we obtain the required estimate.

Proof of Theorem 1.1. In view of Lemma 3.1 it remains to estimate the horospherical distance $d_{b}(\xi, \eta)$ from above. We assume that the points $\xi, \eta \in \partial_{\infty} M \backslash\{\omega\}$ are distinct. Let $u \in \omega \eta, v \in \omega \xi$ be the equiradial points of the triangle $\omega \xi \eta, t=(\xi \mid \eta)_{b}=b(u)=b(v)$. Then $|u v| \leq \delta<1$ by Lemma 2.7. Thus, by Lemma 2.3, we have $d_{E}^{2}(u, v) \leq 17 d_{b, t}(u, v)$, where $d_{b, t}$ is the interior distance on $H_{b, t}$ induced from $M$. By Theorem 2.4, we have $d_{b, t}(u, v) \leq \sinh |u v| \leq \sinh \delta$. Therefore, $d_{E}^{2}(u, v) \leq 17 \sinh \delta=: c_{2}^{2}$. To avoid a standard $\varepsilon$-argument, we assume for simplicity that there is an $E$-horizontal curve $\sigma_{t} \subset H_{b, t}$ between $u, v$ with $\ell_{b, t}\left(\sigma_{t}\right)=d_{E}(u, v)$. The curve $\sigma_{t}$ is the radial projection from $\omega$ of an $E$-horizontal curve $\sigma_{\infty} \subset \partial_{\infty} M \backslash\{\omega\}$ between $\xi, \eta$, and we have

$$
d_{b}(\xi, \eta) \leq \ell_{b}\left(\sigma_{\infty}\right)=e^{-t} \ell_{b, t}\left(\sigma_{t}\right)=e^{-t} d_{E}(u, v) \leq c_{2} e^{-(\xi \mid \eta)_{b}} .
$$

\section{§4. Proof of Theorem 1.2}

We fix $o \in M$ and, for every $t>0$, consider the Riemannian metric $d s_{t}^{2}$ induced from $M$ on the sphere $S_{t} \subset M$ of radius $t$ centered at $o$. Identifying $S_{t}, \partial_{\infty} M$ with the unit 
sphere $U_{o} M \subset T_{o} M$ via the radial projection from $o$, we regard $d s_{t}^{2}$ as the Riemannian metric on $U_{o} M$ for all $t>0$. Then the limit

$$
d s_{\infty}=\lim _{t \rightarrow \infty} e^{-t} d s_{t}
$$

exists, because, given $v \in E_{u}(\lambda)$ with $u \in U_{o} M$, there is a unique Jacobi field $V$ along the geodesic ray $\gamma(t)=\exp _{o} t u, t \geq 0$, with initial data $V(0)=0, \dot{V}(0)=v$. The direction field $V /|V|$ is parallel along $\gamma$ and $|V(t)|=\sinh (t \sqrt{|\lambda|})|v| / \sqrt{|\lambda|}$ for all $t \geq 0$. Thus, $e^{-t} d s_{t}(v)=e^{-t}|V(t)| \rightarrow|v| / 2$ for $\lambda=-1$ and $\rightarrow \infty$ for $\lambda=-4$.

We call the subbundle $E=E(-1) \subset T U_{o} M$ the polarization on the sphere $U_{o} M$. A piecewise smooth curve $\sigma: I \rightarrow U_{o} M$ is said to be E-horizontal if $\dot{\sigma}(t) \in E$ for every $t \in I \subset \mathbb{R}$. Its length is $\ell(\sigma)=\int_{I}|\dot{\sigma}(t)| d t$. We define the distance $d_{E}\left(u, u^{\prime}\right)$ by taking the infimum of the lengths of $E$-horizontal curves between $u, u^{\prime} \in U_{o} M$. This quantity is finite, because for every horosphere $H \subset M$ the canonical embedding $f: H \rightarrow U_{o} M$ is Lipschitz and its differential $d f$ preserves the polarizations on $H, U_{o} M$ by Lemma 2.1 . We choose $\omega \in \partial_{\infty} M$ so that neither $u$ nor $u^{\prime}$ is tangent to $o \omega$, and take $b \in \mathcal{B}(\omega)$ with $b(o)=0$ and $H=b^{-1}(0)$. Then $u, u^{\prime} \in f(H)$, and there is an $E$-horizontal curve $\sigma \subset H$ between $x=f^{-1}(u)$ and $x^{\prime}=f^{-1}\left(u^{\prime}\right)$. Then the curve $f(\sigma) \subset U_{o} M$ is E-horizontal, connects $u$ and $u^{\prime}$, and $\ell_{E}(f(\sigma)) \leq 2 \ell_{E}(\sigma)$ by the proof of Lemma 2.1 .

We denote by $d_{\infty}$ the Carnot-Carathéodory distance on $\partial_{\infty} M$ associated with the Carnot-Carathéodory metric $d s_{\infty}$. Observe that $d s_{\infty}=\frac{1}{2} d s_{E}$ and $d_{\infty}=\frac{1}{2} d_{E}$. Thus, the argument above shows that for every $o \in M, \omega \in \partial_{\infty} M$, and $b \in \mathcal{B}(\omega)$, we have

$$
d s_{\infty} \leq d s_{b} \quad \text { and } \quad d_{\infty} \leq d_{b}
$$

on $\partial_{\infty} M \backslash\{\omega\}$. The following lemma is a minor modification of Lemma 3.1

Lemma 4.1. For each $\xi, \eta \in \partial_{\infty} M$, we have

$$
d_{\infty}(\xi, \eta) \geq c_{1} e^{-(\xi \mid \eta)_{o}}
$$

where $c_{1}=2 e^{-(1+\delta)}$.

Proof. We assume that $\xi \neq \eta$, because otherwise there is nothing to prove. For $\varepsilon>0$ we take an $E$-horizontal curve $\sigma_{\infty} \subset \partial_{\infty} M$ between $\xi$ and $\eta$ with length $\ell_{\infty}\left(\sigma_{\infty}\right) \leq$ $d_{\infty}(\xi, \eta)+\varepsilon$. Let $\sigma_{t} \subset S_{t}$ be its radial projection from $o$. Since the curve $\sigma_{t}$ connects the points $u_{t} \in o \eta, v_{t} \in o \xi$ with $\left|o u_{t}\right|=\left|o v_{t}\right|=t$ and also is $E$-horizontal on $S_{t}$, its length $\ell_{t}\left(\sigma_{t}\right)$ is $2 \sinh t \cdot \ell_{\infty}\left(\sigma_{\infty}\right)$.

Let $u \in o \eta$ and $v \in o \xi$ be the equiradial points of the triangle $o \xi \eta$. Then $t_{0}:=(\xi \mid \eta)_{o}=$ $|o u|=|o v|$. We put $t=t_{0}+1+\delta$. For $u_{t} \in o \eta, v_{t} \in o \xi$ we have $\left|u_{t} v_{t}\right| \geq 2$ by Lemma 2.8 (applied to $o \xi \eta$ ). Thus, $\ell_{t}\left(\sigma_{t}\right) \geq\left|u_{t} v_{t}\right| \geq 2$ and

$$
d_{\infty}(\xi, \eta)+\varepsilon \geq \ell_{\infty}\left(\sigma_{\infty}\right)=\frac{1}{2 \sinh \left(t_{0}+1+\delta\right)} \ell_{t}\left(\sigma_{t}\right) \geq c_{1} e^{-(\xi \mid \eta)_{o}} .
$$

Since $\varepsilon$ has been chosen arbitrarily, we obtain the required estimate.

Lemma 4.2. Assume that points $\xi, \eta, \omega \in \partial_{\infty} M$ are pairwise distinct and that points $v, v^{\prime} \in \xi \omega$ and $w, w^{\prime} \in \xi \eta$ satisfy $b(v)=b(w), b\left(v^{\prime}\right)=b\left(w^{\prime}\right)$ for some and, hence, any Busemann function $b \in \mathcal{B}(\xi)$. Then

$$
\left|v v^{\prime}\right|=\left|w w^{\prime}\right| \leq \ln \frac{\sinh A}{a},
$$

where $A=\max \left\{|v w|,\left|v^{\prime} w^{\prime}\right|\right\}, a=\min \left\{|v w|,\left|v^{\prime} w^{\prime}\right|\right\}$. 
Proof. We put $t=b(v)=b(w), t^{\prime}=b\left(v^{\prime}\right)=b\left(w^{\prime}\right)$ and without loss of generality assume that $t^{\prime}>t$. Let $\sigma_{t^{\prime}} \subset H_{b, t^{\prime}}$ be a shortest curve between $v^{\prime}$ and $w^{\prime}$, i.e., $\ell_{t^{\prime}}\left(\sigma_{t^{\prime}}\right)=$ $d_{b, t^{\prime}}\left(v^{\prime}, w^{\prime}\right)$, and let $\sigma_{t} \subset H_{b, t}$ be the radial projection of $\sigma_{t^{\prime}}$ from $\xi$. Then $e^{t-t^{\prime}} d_{b, t}(v, w) \leq$ $e^{t-t^{\prime}} \ell_{t}\left(\sigma_{t}\right) \leq \ell_{t^{\prime}}\left(\sigma_{t^{\prime}}\right)=d_{b, t^{\prime}}\left(v^{\prime}, w^{\prime}\right)$. We have $d_{b, t}(v, w) \geq a$, and $d_{b, t^{\prime}}\left(v^{\prime}, w^{\prime}\right) \leq \sinh A$ by Theorem 2.4. Thus, $\left|v v^{\prime}\right|=\left|w w^{\prime}\right|=t^{\prime}-t \leq \ln \frac{\sinh A}{a}$.

Lemma 4.3. Assume that $(\xi \mid \eta)_{o} \geq 1+\delta$ for some points $o \in M$ and $\xi, \eta \in \partial_{\infty} M$. Then for $\omega \in \partial_{\infty} M$ opposite to $\xi$ with respect to o, i.e., $o \in \omega \xi$, and the function $b \in \mathcal{B}(\omega)$ with $b(o)=0$, we have

$$
\left|(\xi \mid \eta)_{b}-(\xi \mid \eta)_{o}\right| \leq c_{3}=\ln \frac{\sinh (2+3 \delta)}{2} .
$$

Proof. We can assume that $\xi \neq \eta$, because otherwise $(\xi \mid \eta)_{b}=(\xi \mid \eta)_{o}=\infty$. Furthermore, we have $(\xi \mid \omega)_{o}=0$, so that $\eta \neq \omega$, and $\omega, \xi, \eta$ are pairwise distinct.

Let $v \in o \xi, u \in o \eta, w \in \xi \eta$ be the equiradial points of the triangle $o \xi \eta$. Then $|o v|=(\xi \mid \eta)_{o} \geq 1+\delta$, and thus, there is $v^{\prime} \in o v$ such that $\left|v v^{\prime}\right|=1+\delta$. Also, there is $w^{\prime} \in w \eta$ such that $\left|w w^{\prime}\right|=1+\delta$. Then $\left|v^{\prime} w^{\prime}\right| \leq 2+3 \delta$. On the other hand, arguing as in Lemma 2.8, we obtain $\left|v^{\prime} w^{\prime}\right| \geq 2$.

Applying a similar argument to the equiradial points $\widetilde{v} \in \xi \omega, \widetilde{w} \in \xi \eta, \widetilde{u} \in \omega \eta$ of the triangle $\xi \eta \omega$, we see that

$$
2 \leq\left|v^{\prime \prime} w^{\prime \prime}\right| \leq 2+3 \delta
$$

for $v^{\prime \prime} \in \xi \omega, w^{\prime \prime} \in \xi \eta$ with $b^{\prime}\left(v^{\prime \prime}\right)=b^{\prime}\left(w^{\prime \prime}\right)=(\eta \mid \omega)_{b^{\prime}}+1+\delta$ for any Busemann function $b^{\prime} \in \mathcal{B}(\xi)$.

Then $\left|v^{\prime} v^{\prime \prime}\right| \leq \ln \frac{\sinh (2+3 \delta)}{2}$ by Lemma 4.2, It remains to observe that $\left|(\xi \mid \eta)_{b}-(\xi \mid \eta)_{o}\right|=$ $|v \widetilde{v}|=\left|v^{\prime} v^{\prime \prime}\right|$, because $b(o)=0$ and $(\xi \mid \eta)_{b}=b(\widetilde{v})=|o \widetilde{v}|$.

Recall that $d_{\infty}$ is the spherical metric on $\partial_{\infty} M$ centered at $o \in M$.

Lemma 4.4. If $\xi, \eta \in \partial_{\infty} M$ and $d_{\infty}(\xi, \eta) \leq 2 e^{-(2+\delta)}$, then $(\xi \mid \eta)_{o} \geq 1+\delta$.

Proof. Let $\xi_{t} \in o \xi$ and $\eta_{t} \in$ on be points with $\left|o \xi_{t}\right|=\left|o \eta_{t}\right|=t$ for every $t \geq 0$. Recall that $d s_{t}(v)=2 \sinh t \cdot d s_{\infty}(v)$ for every $v \in E \subset T U_{o} M$. Therefore, $\left|\xi_{t} \eta_{t}\right| \leq d_{t}\left(\xi_{t}, \eta_{t}\right) \leq$ $e^{t} d_{\infty}(\xi, \eta)$. Using the monotonicity of the Gromov product, we obtain

$$
(\xi \mid \eta)_{o} \geq\left(\xi_{t} \mid \eta_{t}\right)_{o}=t-\frac{1}{2}\left|\xi_{t} \eta_{t}\right| \geq t-e^{t-(2+\delta)}=1+\delta
$$

for $t=2+\delta$.

Proposition 4.5. For every $\xi, \eta \in \partial_{\infty} M$ with $d_{\infty}(\xi, \eta) \leq 2 e^{-(2+\delta)}$, we have

$$
d_{\infty}(\xi, \eta) \leq c_{2}^{\prime} e^{-(\xi \mid \eta)_{o}}
$$

where $c_{2}^{\prime}=c_{2} e^{c_{3}}$ and $c_{2}$ is the constant from Theorem 1.1 .

Proof. By Lemma 4.4, we have $(\xi \mid \eta)_{o} \geq 1+\delta$. Take $\omega \in \partial_{\infty} M$ opposite to $\xi$ with respect to $o$, and let $b \in \mathcal{B}(\omega), b(o)=0$. Then $(\xi \mid \eta)_{b} \geq(\xi \mid \eta)_{o}-c_{3}$ by Lemma 4.3 and using Theorem 1.1] we obtain

$$
d_{\infty}(\xi, \eta) \leq d_{b}(\xi, \eta) \leq c_{2} e^{-(\xi \mid \eta)_{b}} \leq c_{2}^{\prime} e^{-(\xi \mid \eta)_{o}} .
$$

Proof of Theorem 1.2. Let $D=\operatorname{diam} \partial_{\infty} M$ be the diameter of $\partial_{\infty} M$ with respect to (any) spherical metric $d_{\infty}$ (for $M=\mathbb{K} \mathrm{H}^{n}$, this is clearly independent of $\mathbb{K}=\mathbb{C}, \mathbb{H}, \mathbb{C} a$ and $n \geq 2$; cf. Lemma 2.3). We show that

$$
d_{\infty}\left(\xi, \xi^{\prime}\right) \leq c_{2}^{\prime \prime} e^{-\left(\xi \mid \xi^{\prime}\right)_{o}}
$$

for each $\xi, \xi^{\prime} \in \partial_{\infty} M$, where $c_{2}^{\prime \prime}=\max \left\{D e^{1+\delta}, c_{2}^{\prime}\right\}$. 
If $\left(\xi \mid \xi^{\prime}\right)_{o} \geq 1+\delta$, then we argue as in Proposition 4.5 obtaining $d_{\infty}\left(\xi, \xi^{\prime}\right) \leq c_{2}^{\prime} e^{-\left(\xi \mid \xi^{\prime}\right)_{o}}$. This allows us to assume that $\left(\xi \mid \xi^{\prime}\right)_{o}<1+\delta$. Then

$$
d_{\infty}\left(\xi, \xi^{\prime}\right) \leq D \leq D e^{1+\delta} e^{-\left(\xi \mid \xi^{\prime}\right)_{o}} .
$$

The estimate $d_{\infty}\left(\xi, \xi^{\prime}\right) \geq c_{1} e^{-\left(\xi \mid \xi^{\prime}\right)_{o}}$ was obtained in Lemma 4.1

\section{REFERENCES}

[Bo] M. Bourdon, Structure conforme au bord et flot géodésique d'un CAT(-1)-espace, Enseign. Math. (2) 41 (1995), 63-102. MR1341941 (96f:58120)

[BS] S. Buyalo and V. Schroeder, Elements of asymptotic geometry, EMS Monogr. in Math., European Math. Soc. (EMS), Zürich, 2007. MR2327160(2009a:53068)

[FS] T. Foertsch and V. Schroeder, Hyperbolicity, CAT(-1)-spaces and the Ptolemy inequality, arXiv:math/0605418v2.

[HI] E. Heintze and H.-C. Im Hof, Geometry of horospheres, J. Differential Geom. 12 (1977), 481-491. MR0512919 (80a:53051)

$[\mathrm{Ku}]$ A. Kuznetsov, Visibility metrics on the boundary at infinity for the complex hyperbolic plane, Zap. Nauchn. Sem. S.-Peterburg. Otdel. Mat. Inst. Steklov. (POMI) 353 (2008), 70-92; English transl. in J. Math. Sci. (New York) (to appear).

[Mo] G. D. Mostow, Strong rigidity of locally symmetric spaces, Ann. of Math. Stud., No. 78, Princeton Univ. Press, Princeton, NJ, 1973. MR0385004 (52:5874)

[Pa] P. Pansu, Métriques de Carnot-Carathéodory et quasiisométries des espaces symétriques de rang un, Ann. of Math. (2) 129 (1989), 1-60. MR0979599 (90e:53058)

[Wo] J. Wolf, Spaces of constant curvature, Univ. of California, Berkeley, CA, 1972. MR0343213 (49:7957)

St. Petersburg Branch, Steklov Institute of Mathematics, Fontanka 27, St. Petersburg 191023, RUSSIA

E-mail address: sbuyalo@pdmi.ras.ru

Department of Mathematics and Mechanics, St. Petersburg State University, Universitetskil Prospekt 28, Staryi Petergof, St. Petersburg 198504, Russia

E-mail address: kasheftin@gmail.com

Received 20/MAY/2009

Translated by THE AUTHORS 